À propos d'un ouvrage peu connu dans la sociologie française : Making it count. The improvement of social research and theory de Stanley Lieberson

Louis-André Vallet

\title{
CpenEdition
}

Journals

Édition électronique

URL : http://journals.openedition.org/ress/441

DOI : $10.4000 /$ ress.441

ISSN : 1663-4446

Éditeur

Librairie Droz

Édition imprimée

Date de publication : 1 mars 2004

Pagination : 341-348

ISBN : 2-600-00941-8

ISSN : 0048-8046

Référence électronique

Louis-André Vallet, «À propos d'un ouvrage peu connu dans la sociologie française: Making it count. The improvement of social research and theory de Stanley Lieberson », Revue européenne des sciences sociales [En ligne], XLII-129 | 2004, mis en ligne le 06 novembre 2009, consulté le 03 mai 2019. URL http://journals.openedition.org/ress/441 ; DOI : 10.4000/ress.441 


\section{À PROPOS D'UN OUVRAGE PEU CONNU DANS LA SOCIOLOGIE FRANÇAISE : Making it count. The improvement of social research and theory de Stanley Lieberson}

Il est probablement assez peu fréquent que les volumes de Mélanges publiés in memoriam d'un auteur et d'un collègue trop tôt disparu fassent explicitement mention des débats scientifiques, éventuellement vifs, dans lesquels celui-ci a pu être impliqué. Au risque de surprendre, mais en espérant ne pas choquer, c'est pourtant le point de départ que j'adopterai dans cette contribution en évoquant le vif échange paru en 1995 dans American Journal of Sociology entre Philippe Besnard et Stanley Lieberson; il faisait suite à un article de Lieberson et d'Eleanor O. Bell paru trois ans plus tôt dans la même revue. Dans l'ensemble des travaux que Philippe Besnard a consacrés à la formation sociale des goûts - et, plus précisément, au choix des prénoms comme à l'étude de leur diffusion, au cours du temps et entre les groupes sociaux - son commentaire initial et la réplique vive de Lieberson qui l'a suivi ne sont probablement qu'un épiphénomène dont il ne faut pas majorer l'importance. Ils constituent sans doute un bon indicateur d'une époque - qui n'est pas si lointaine, mais dont on voudrait espérer qu'elle soit aujourd'hui révolue - où, pour parler vite, «la sociologie française» reprochait à «la sociologie américaine» de ne pas suffisamment la lire ou la citer, alors que la seconde répliquait en laissant nettement entendre que la première lui semblait sous-estimer le degré d'exigence méthodologique et de précision dans l'analyse des données devenu commun dans les revues d'outre-Atlantique. Sans doute n'est-il guère utile de s'appesantir davantage sur ce type de malentendu.

Cependant et au risque de paraître paradoxal, je voudrais saisir l'occasion de cette contribution pour soutenir la thèse selon laquelle les deux protagonistes du débat sont en réalité plus proches que leur querelle ne le laisse supposer et qu'ils ont en commun le fort intérêt et la grande importance que chacun d'eux accorde aux questions de méthode en sciences sociales, spécialement aux questions de méthodologie quantitative. Pour étayer cette thèse, il ne sera pas nécessaire de revenir ici sur la centralité de ces aspects pour le spécialiste français de sociologie durkheimienne. Je développerai en revanche ce qu'il en est du professeur de Berkeley, puis d'Harvard - et du président de l'Association Américaine de Sociologie pour l'année 1991 - en évoquant l'un de ses ouvrages, paru en 1985, mais qui, à ma connaissance, est resté peu connu en France - une recherche simple montre par exemple qu'il n'est présent que dans deux bibliothèques universitaires de l'hexagone.

Écrit par un sociologue engagé dans la recherche empirique depuis de nombreuses années, Making it count. The improvement of social research and 
theory se présente comme une critique raisonnée de la logique et des usages qui sous-tendent la recherche quantitative contemporaine en sciences sociales. L'ouvrage est d'ailleurs divisé en deux parties, «Pratiques actuelles» et «Vers une solution», et emprunte ses exemples à la sociologie comme à la science sociale appliquée. Dès le chapitre introductif et en reconnaissant que les chercheurs ont parfois la prétention de s'attaquer à des tâches «infaisables » (undoable), l'auteur affirme explicitement une conception modeste de la recherche empirique: certaines questions de recherche sont intrinsèquement impossibles à traiter avec les techniques des sciences sociales, d'autres sont prématurées compte tenu de l'état actuel de la connaissance, d'autres encore sont trop compliquées et il y a enfin des questions à propos desquelles la connaissance empirique et théorique a pu mettre en évidence qu'elles étaient vaines.

La thèse centrale de l'ouvrage peut être résumée ainsi: alors que la majeure partie de la recherche en sociologie est fondée sur des données non expérimentales - situation compréhensible compte tenu des limites imposées par la société le modèle expérimental n'occupe pas une place mineure dans le développement des procédures de recherche; au contraire, les données non expérimentales - la source normale d'information empirique - sont traitées autant que possible comme si elles étaient issues d'expérimentations contrôlées. À l'appui de cette thèse, Lieberson invoque notamment une citation particulièrement claire de Burgess en 1929. L'ouvrage va donc viser à mettre en évidence qu'en adoptant une telle posture, les chercheurs s'exposent à fonder leurs raisonnements sur des hypothèses irréalistes et, par là, à conduire des analyses comme à tirer des conclusions sujettes à caution ou erronées. Il va aussi s'efforcer de dégager une alternative sur laquelle fonder les efforts en vue d'une science sociale rigoureuse, capable d'utiliser l'évidence empirique pour évaluer les théories et les propositions développées à propos de la société.

Le chapitre II ( $«$ Selectivity») doit probablement être considéré comme le cœur de l'ouvrage. Les sciences sociales traitent continuellement de situations dans lesquelles les sujets n'ont pas été affectés aléatoirement aux différentes conditions d'observation. En d'autres termes, un processus sélectif intervient qui, en luimême, est susceptible d'influencer la variable dépendante ou le résultat observé dans les diverses conditions d'étude. Le problème typique du chercheur est donc de ne pas savoir si le résultat observé (par exemple, les différences de performance scolaire entre élèves fréquentant des écoles publiques et élèves fréquentant des écoles privées) reflète purement et simplement les forces prises en considération (ici, le fait qu'il existerait une différence d'efficacité entre les deux types d'école), ou s'il renvoie aussi, à un certain degré, à des différences non mesurées entre les populations qui caractérisent les diverses conditions (par exemple, le fait que la répartition selon l'origine socio-économique ou le niveau d'ambition n'est éventuellement pas la même dans les deux types d'école). Ce problème très général de sélectivité a conduit à la démarche méthodologique du «contrôle des variables » (via une analyse de régression, un tri croisé approprié, ou toute autre technique équivalente). En prenant en compte dans l'analyse les différences entre populations caractéristiques des diverses conditions (par exemple, en «contrôlant» l'origine socio-économique, le niveau d'ambition, etc.), le chercheur interprète le résultat relatif aux écoles (l'écart de performance entre élèves des écoles publiques et des écoles privées, net de l'influence des autres variables) comme s'il 
reflétait le résultat qui aurait été observé si une véritable expérimentation avait été mise en place.

Qu'est-ce qui pose problème dans une telle procédure? La raison conduisant à tenir compte des différences qui peuvent exister entre les populations - le fait que les processus sociaux sont des processus sélectifs - est simultanément celle qui doit conduire à douter qu'un tel effort de «contrôle» puisse être bien souvent couronné de succès. Car il est probable que des processus non aléatoires d'affectation des sujets opèrent encore à l'intérieur des variables de contrôle ellesmêmes. Bref, ce qui est en jeu, c'est la possible incomplétude de la procédure de contrôle. Les chercheurs en sciences sociales reconnaissent volontiers cette éventualité, mais la plupart d'entre eux se comportent comme si l'application, même partielle, de contrôles constituait une procédure bénéfique ou, à la limite, inoffensive. En d'autres termes, les sociologues sont très majoritairement enclins à penser que le résultat net obtenu après prise en compte de (certaines) variables de contrôle est plus proche - ou, au pire, n'est pas moins proche - de la relation «vraie» entre variable d'intérêt et variable dépendante - celle que fournirait une approche rigoureusement expérimentale - que ce n'était le cas du résultat brut initialement observé.

Or, comme l'affirme Lieberson avec force, il n'en va pas nécessairement ainsi. Sous certaines conditions, la «sélectivité non mesurée », encore appelée hétérogénéité non observée, peut au contraire conduire à ce que la procédure de contrôle engendre un résultat plus éloigné de la relation vraie et parfois opposé à celle-ci. L'auteur l'illustre clairement à partir de l'exemple de la performance scolaire selon le type d'école fréquenté : examinant tous les cas qui résultent d'une part de l'existence éventuelle et de la direction du lien entre variable de contrôle (l'origine socio-économique) et variable dépendante, d'autre part de l'existence éventuelle d'une sélectivité à l'intérieur de la variable de contrôle et de la direction dans laquelle elle opère, il met en évidence que le raisonnement quasi expérimental conduit parfois à une conclusion qui éloigne de la réalité. De même, dans une situation où la variable d'intérêt (le type d'école) n'aurait rigoureusement aucune influence sur la variable dépendante (la performance des élèves), l'impact d'une sélectivité non mesurée est susceptible de prendre la forme d'un effet apparent du type d'école! Ou encore, il est fréquent qu'une variable, disposant des mêmes propriétés formelles dans deux situations, varie en réalité dans ses conséquences en raison des différences qu'elle masque sur d'autres attributs - par exemple, entre deux régions où la distribution du niveau d'éducation est très différente, le fait de contrôler cette variable, c'est-à-dire de raisonner à niveau d'éducation égal, peut conduire à comparer des individus très différents du point de vue de leurs capacités ou de leur niveau d'ambition.

En soulignant qu'il constitue l'un des éléments les plus routiniers, mais aussi les plus acceptés de la logique des sciences sociales, l'auteur revient encore sur le contrôle des variables dans le chapitre VI. La conclusion qu'il en tire est radicale et diamétralement opposée à la pratique habituelle. Dans la recherche quasi expérimentale, il est vraisemblable que l'usage d'une procédure de contrôle n'est approprié que lorsqu'elle s'avère en définitive inutile, c'est-à-dire lorsque la prise en compte de la variable de contrôle $\mathrm{X}_{2}$ n'altère pas le résultat initialement observé quant à l'influence de $\mathrm{X}_{1}$ sur $\mathrm{Y}$. Cela constitue en effet un bon signe du fait qu'une approximation du modèle expérimental et de l'affectation 
aléatoire des individus aux conditions peut raisonnablement être maintenue. En revanche, si la relation initiale est significativement altérée après que l'influence d'une variable de contrôle $X_{2}$ a été prise en compte, on ne peut habituellement immédiatement conclure que la technique du contrôle nous a rapproché de l'influence «vraie» de $\mathrm{X}_{1}$ sur $\mathrm{Y}$. Au contraire, la conclusion doit être méthodologique plutôt que substantielle: il existe une relation statistique entre $X_{1}$ et $X_{2}$, et il convient d'examiner plus avant cette relation - il faut en particulier savoir pourquoi elle existe - pour déterminer si elle est ou non sous-tendue par un processus sélectif susceptible d'invalider l'analogie avec le modèle expérimental. En d'autres termes, alors que les chercheurs se centrent habituellement sur les relations qui existent entre les variables $\mathrm{X}_{1}, \mathrm{X}_{2}, \mathrm{X}_{3} \ldots$ et $\mathrm{Y}$, Lieberson nous invite instamment à accorder davantage d'importance aux liens qui unissent les premières. Par exemple, s'il existe une relation entre l'origine socio-économique $\left(\mathrm{X}_{2}\right)$ et le fait de fréquenter une école privée $\left(\mathrm{X}_{1}\right)$, se pourrait-il que d'autres aspects inobservés différencient, parmi les élèves d'un milieu socioéconomique donné, ceux qui fréquentent des écoles privées de ceux qui sont scolarisés dans des écoles publiques? Si oui, ces facteurs affectent-ils également la variable dépendante Y (la performance scolaire)? Si tel est le cas et en l'absence de leur prise en compte explicite, il s'avère impossible d'évaluer correctement l'influence du type d'école et de l'origine socio-économique sur la performance des élèves.

La question de la sélectivité n'est pas le seul problème que doit affronter la recherche empirique quasi-expérimentale en sociologie. Celle-ci est, sous une forme ou sous une autre, une recherche comparative et, selon Stanley Lieberson, les comparaisons mises en jeu sont souvent irrémédiablement rendues caduques par quatre pratiques courantes: le problème de contamination et l'erreur qu'il est susceptible d'engendrer (chapitre III); l'hypothèse d'une causalité réversible (chapitre IV); la confusion des niveaux d'analyse (chapitre V) et l'usage abusif du critère de la variance expliquée pour décider ce qu'il convient d'étudier et l'interprétation que l'on peut en donner (chapitre V).

Un problème de contamination survient lorsque l'impact d'une variable indépendante n'est pas limité aux seules situations dans lesquelles elle est présente. Il s'ensuit que, dans un tel cas, l'influence de cette variable ne peut être correctement déterminée à travers la méthode comparative et le chercheur qui procéderait ainsi s'exposerait à commettre l'erreur de contamination. Supposons par exemple que l'on souhaite déterminer l'effet de l'entrée de la Norvège dans la Seconde Guerre mondiale sur la fécondité dans ce pays. Pour ce faire, il pourrait sembler très raisonnable de procéder à une comparaison avec la Suède, pays voisin, mais resté neutre au cours du conflit. Comme l'indique Lieberson, on commettrait alors l'erreur de contamination car une étude a pu montrer de manière très convaincante qu'en dépit de la neutralité du pays la fécondité suédoise avait varié, durant cette période, en raison directe des épisodes marquants du conflit. Il est probable que le problème de contamination soit très répandu aujourd'hui du fait de l'intense communication qui existe entre les sociétés et, à l'intérieur d'une société, entre ses différents segments sociaux et spatiaux. De même, chaque fois qu'un processus social met en jeu, sous une forme ou sous une autre, une anticipation de certains événements ou bien une réaction à de tels événements survenus ailleurs, alors l'erreur de contamination est susceptible d'exister. 
Si un changement dans le niveau d'une certaine variable $X_{1}$ est responsable d'une variation d'une autre variable $Y$, que va-t-il advenir d'Y si $X_{1}$ retourne à son niveau initial? En supposant toute chose égale par ailleurs, le processus sera qualifié de réversible si Y revient elle-même à sa valeur de départ et d'irréversible si tel n'est pas le cas - on peut encore distinguer entre un processus totalement irréversible et un processus partiellement irréversible. Or, la recherche en sciences sociales tend généralement à supposer que les processus sociaux sont parfaitement réversibles et à négliger les formes asymétriques de causalité: si une variation de $\mathrm{X}_{1}$ a pour conséquence un changement de $\mathrm{Y}$ dans une certaine direction, alors certainement la variation opposée de $\mathrm{X}_{1}$ engendrera un changement de $\mathrm{Y}$ de même nature. Pourtant, cette distinction entre processus réversibles et irréversibles est aussi cruciale pour la recherche appliquée que pour la recherche fondamentale. Supposons en effet que le processus causal responsable d'un problème social soit totalement ou largement irréversible. Le fait de ne pas le reconnaître conduira à l'échec toute politique qui viserait à résoudre le problème en supprimant la cause initiale - une solution qui ne peut être efficace que si le processus est parfaitement réversible. Bien plus, cela peut avoir pour conséquence de remettre en cause un diagnostic initialement valide sur les causes véritables du problème: puisque celui-ci ne disparaît pas lorsque les variables influentes sont ramenées à leur niveau initial, le chercheur peut être incité à conclure, de manière erronée, qu'elles n'en constituaient pas les déterminants essentiels !

La quasi-totalité des chercheurs en sciences sociales souhaitent disposer de données qui manifestent une certaine variabilité dans les caractéristiques auxquelles ils s'intéressent. À partir de là, la croyance commune est que les théories existantes seront validées et que de nouvelles théories pourront être développées en déterminant quels critères permettent de rendre compte de cette variation observée. Or, selon l'auteur, il existe une tendance fâcheuse à ériger la «variance expliquée» comme un but en soi et cela conduit à privilégier certaines questions de recherche comme à en ignorer d'autres. Comme l'affirme Lieberson avec force, il n'est pas nécessairement raisonnable, ni même désirable, qu'une théorie vise à rendre compte de la plus grande quantité possible de variance d'une variable dépendante. De même, il est faux de croire que l'importance, absolue ou relative, de diverses forces causales pourrait être rigoureusement déterminée à partir de la quantité de variance qu'elles «expliquent» dans le cadre d'une étude empirique. Par ailleurs, ce n'est pas la même chose d'expliquer un phénomène - par exemple, le statut socio-économique - que d'expliquer les variations qui caractérisent ce phénomène - ici, les différences inter-individuelles de statut socio-économique - et la seconde tâche ne peut être tenue pour équivalente à la première. Au contraire, nous devrions nous satisfaire de commencer par comprendre pourquoi une entité ou un processus social existe avant de songer à aborder les questions plus mineures relatives à ses variations. La centration sur le critère de la variance expliquée a enfin pour conséquence négative que l'on pose de façon privilégiée les problèmes de recherche à un certain niveau d'analyse - usuellement, le niveau individuel qui est celui où la variabilité s'exprime le plus. Il s'ensuit fréquemment une confusion des niveaux d'analyse qui mine la capacité du chercheur à évaluer correctement les théories existantes ou à produire des conclusions valides relatives aux causes de certains phénomènes: si une proposition théorique est formulée à un certain niveau d'analyse, alors l'évidence 
empirique obtenue à un niveau inférieur ne pourra être pertinente pour déterminer le degré de validité de cette proposition.

La première partie de l'ouvrage, centrée sur les «pratiques actuelles», s'achève par un chapitre complémentaire où l'auteur insiste notamment sur le lien, insuffisamment perçu à ses yeux, entre les objectifs de recherche et l'analyse empirique mise en œuvre. Plusieurs objectifs différents peuvent être poursuivis - de la découverte de faits aux recommandations en matière de politique à mettre en œuvre, en passant par l'explication d'un ensemble de faits, puis l'évaluation d'une théorie - auxquels correspondent ordinairement des formes d'analyse empirique distinctes, conduites sur des ensembles de données qui ne sont pas nécessairement les mêmes. Ainsi par exemple, les données nécessaires pour une recherche appliquée orientée vers le traitement d'une situation sont généralement différentes de celles à partir desquelles peuvent être déterminées les causes de la situation en question et il n'est guère possible de tirer des conclusions de la seconde entreprise des recommandations pour la première.

Après avoir argumenté que plusieurs des procédures les plus largement acceptées dans la recherche empirique contemporaine en sociologie reposaient sur des modèles et des hypothèses largement inappropriées et que leur application était souvent contre-productive, Stanley Lieberson avance, dans les derniers chapitres, quelques pistes de solution qui n'abandonneraient pas l'objectif de fonder une «science rigoureuse de la société » (p. 171). Dans le chapitre IX intitulé «Repenser la causalité », l'auteur affirme tout d'abord que les sociologues théoriciens comme les spécialistes de recherche empirique ne peuvent plus continuer à négliger la distinction entre les formes symétriques et asymétriques de la causalité, puis il se livre à un vigoureux plaidoyer en faveur de l'utilisation de données longitudinales. Son argument essentiel réside ici dans le fait que les données transversales (cross-sectional) recueillies à une date unique ne peuvent très généralement fournir une base solide pour l'analyse causale - on rappellera que l'ouvrage a été publié en 1985 et que, depuis environ une décennie, le vœu de l'auteur d'une utilisation accrue de données longitudinales est en voie d'être exaucé. Enfin, face à la multiplication et à l'émiettement des causes que suggère souvent l'application de l'analyse multivariée aux phénomènes sociaux, Lieberson élabore une distinction entre causes superficielles et causes fondamentales qui est en même temps une critique des interprétations trop immédiates de l'analyse de régression. Par exemple, le différentiel d'éducation entre Noirs et Blancs aux États-Unis pourra être considéré comme une cause fondamentale du différentiel de revenu entre les deux races si, indépendamment de tout autre facteur, une variation du premier a pour résultante une transformation du second. En revanche, le différentiel d'éducation ne sera qu'une cause superficielle si sa variation - par exemple, sa réduction - n'affecte pas en réalité le niveau du différentiel de revenu, mais n'a pour conséquence que d'altérer le poids de l'éducation dans la détermination du revenu et/ou, éventuellement, d'accroître l'importance apparente d'une autre cause superficielle. Ainsi, même si une analyse de régression établie à un instant donné fait apparaître que le différentiel d'éducation «explique», en un sens statistique, $x \%$ du différentiel de revenu, il ne faut pas s'attendre, si la première variable n'est qu'une cause superficielle de la seconde, à ce que la mise en œuvre d'une réduction réelle de l'écart d'instruction entre les races affecte en quoi que ce soit l'écart de revenu qui les sépare. En effet, il est fort probable que 
la réduction du différentiel d'éducation s'accompagnera d'un réaménagement de l'économie d'ensemble des causes superficielles dont le résultat essentiel consistera en la stabilité de l'écart initialement observé pour la variable dépendante. Il est alors possible que la cause fondamentale du différentiel de revenu reste une cause non observée - ici, la discrimination raciale. Pour pouvoir être précisément établie, la distinction entre causes fondamentales et causes superficielles nécessite en réalité d'observer et de mettre en relation, au cours du temps, les changements intervenus dans la composition des populations, les transformations de la variable dépendante et de ses liens avec les causes potentielles.

Enfin, dans le chapitre X («From controls to outcomes»), l'auteur revient une nouvelle fois sur la procédure du contrôle des variables, si habituelle et routinière dans la sociologie nord-américaine. Après avoir précisé dans quelles situations un tel usage était ou n'était pas approprié, Lieberson reconnaît que certains développements intervenus en statistique et économétrie au tournant des années quatrevingt ont eu précisément pour objet de traiter la question difficile des biais de sélection, mais il exprime aussi ses doutes quant au degré de succès de ces tentatives. Il souligne également que l'échec de la procédure du contrôle des variables tient fréquemment au fait que ce dernier porte, de façon privilégiée, sur les causes superficielles qui n'entretiennent donc pas un rapport fondamental avec la variable dépendante. Insistant sur le fait qu'il n'est pas nécessaire que les sciences sociales «simulent des expériences» pour qu'elles puissent être considérées comme des sciences, Stanley Lieberson suggère enfin aux chercheurs d'accorder moins d'importance aux causes superficielles et au contrôle des variables pour centrer davantage leur attention sur les faits qu'il s'agit d'expliquer et sur la recherche de leurs causes fondamentales.

Au moment de sa publication, Making it count a retenu l'attention dans la sociologie américaine. Le livre a fait l'objet de compte rendus dans les revues de premier plan - par exemple American Journal of Sociology, Contemporary Sociology ou Social Forces - et Sociological Methodology lui a aussi consacré deux contributions. L'ouvrage a, semble-t-il, été reçu diversement. Certains ont souligné qu'il avait pour résultat positif de forcer la sociologie quantitative à revenir à ses «fondamentaux ». Mais il a par exemple été reproché à l'auteur que les solutions proposées dans la seconde partie n'apparaissaient pas à la hauteur des critiques émises ou encore qu'il n'avait jamais explicitement défini les notions «d'expérience» et de «quasi-expérience» qu'il emploie si souvent. On a également souligné qu'il n'était pas au fait des derniers développements techniques relatifs aux biais de sélection et aux erreurs de spécification des modèles. Il reste qu'aujourd'hui encore et pour le lecteur français, Making it count peut être lu comme une critique lucide et stimulante des limites que rencontrait - et rencontre toujours en partie aujourd'hui - la sociologie américaine, empirique et quantitative, une critique menée «de l'intérieur» par l'un de ses promoteurs. Un livre, en définitive, dont je ne crois pas que Philippe Besnard avait connaissance, mais dont je pense sincèrement qu'il l'aurait apprécié.

Laboratoire de sociologie quantitative, CNRS et CREST, Paris louis-andre.vallet@wanadoo.fr 


\section{RÉFÉRENCES BIBLIOGRAPHIQUES}

Arminger G., Bohrnstedt G. W., 1987, «Making it count even more: a review and critique of Stanley Lieberson's Making it count. The improvement of social theory and research», Sociological Methodology, 17, pp. 363-372.

Berk R. A., 1986, Review of Making it count. The improvement of social research and theory, American Journal of Sociology, 92(2), pp. 462-465.

Besnard Ph., 1995, «The study of social taste through first names: comment on Lieberson and Bell», American Journal of Sociology, 100(5), pp. 1313-1317.

Campbell R. T., 1987, Review of Making it count. The improvement of social research and theory, Social Forces, 65(3), pp. 905-906.

Costner H. L., 1986, Review of Making it count. The improvement of social research and theory, Contemporary Sociology, 15(4), pp. 537-540.

Lieberson S., 1985, Making it count. The improvement of social research and theory, Berkeley, University of California Press.

- 1995, «Reply to Philippe Besnard», American Journal of Sociology, 100(5), pp. 1317-1325.

- Bell E. O., 1992, «Children's first names: an empirical study of social taste», American Journal of Sociology, 98(3), pp. 511-554.

Singer B., Marini M. M., 1987, «Advancing social research: an essay based on Stanley Lieberson's Making it count», Sociological Methodology, 17, pp. 373-391. 